\title{
Design and Implementation of English Writing Evaluation System
}

\section{Weiwei Qu}

Teaching and Research Institute of Foreign Languages, Bohai University, Jinzhou, 121013, China

syquwei@yeah.net

Keywords: English writing; evaluation system; design and implementation; design thought; system function; key technology

\begin{abstract}
Writing assessment is an important part of writing teaching, which directly affects students' writing motivation and writing attitude. The traditional evaluation method of College English writing is single, and the effectiveness of the evaluation is not enough to improve the students' writing ability. Multiple Intelligence theory advocates students to participate in the writing evaluation process, and advocates the pluralism of evaluation subject. In order to improve the information level of English writing assessment, this paper designs three methods, which are automatic evaluation, automatic evaluation, automatic evaluation and student evaluation. The design of the system is based on the hierarchical structure and the object-oriented design idea. Based on the principles of abstraction, incremental refinement, modular system and information concealment, the system is designed based on MS.NET platform.
\end{abstract}

\section{Introduction}

Evaluation of English Writing is an Important Link in College English Writing Teaching. English writing is a complex and repetitive cognitive process, writing evaluation should run through the whole process of writing. In the current evaluation of college English writing, teachers are the main body of the evaluation, the students writing to complete the task of teacher placement, the teacher is the student works the only reader, a single evaluation means closed. Teachers pay more attention to grammatical errors, lexical errors and sentence structure errors, and the validity of the evaluation is not enough to measure the students' learning achievement. Multiple Intelligence theory is concerned with the improvement of students' writing ability, advocates students to participate in the process of writing evaluation, advocates the diversification of the subject of evaluation, evaluation and evaluation, teachers and students, and forms a kind of "interaction subject" Is no longer the negative coping of the evaluation, but the active participants in the evaluation [1,2]. In order to improve the information level of English writing evaluation research, assisted by software system evaluation, including automatic evaluation and automatic evaluation and evaluation of students in three ways, each also includes a specific evaluation module, convenient for teachers and students to use.

\section{System Design Thought}

The design thought is the thinking mode and logical mode of the designer in the process of design and design. In order to improve the system stability, easy operation, maintenance and safety, to achieve the design target of the system of English writing objective and accurate, to make system design idea from hierarchical structure design and object-oriented design.

(1) Multi layer structure design. As an important symbol of human civilization and progress, social division of labor makes each individual in the society under the condition of limited resources can only focus on the specific work, but also can exchange work with others, which greatly improves the labor efficiency. The idea of division of labor also runs through the history of the development of software, that is, subroutine, function process, object, component, layer". The division of labor is more and more complete, the level of abstraction is higher and higher, the granularity of the model is more and more big, and the distance from the real world is getting closer and closer. In the software system structure design, there is also the idea of this division of labor, that is, hierarchical structure. Well known hierarchies such as open system interconnection 
reference model. The characteristics of the hierarchical structure is used to provide services to the lower level, and only through specific interface to obtain lower level ask service, provide specific services for the upper layer through a specific interface, and does not depend on the upper level, also do not know the existence of the upper. There is one to many relationship between the lower layer and the adjacent upper layer, that is to say, the same lower layer may provide services for different layers. The system is based on the design and development of multi layer logic structure, as shown in Fig. 1.

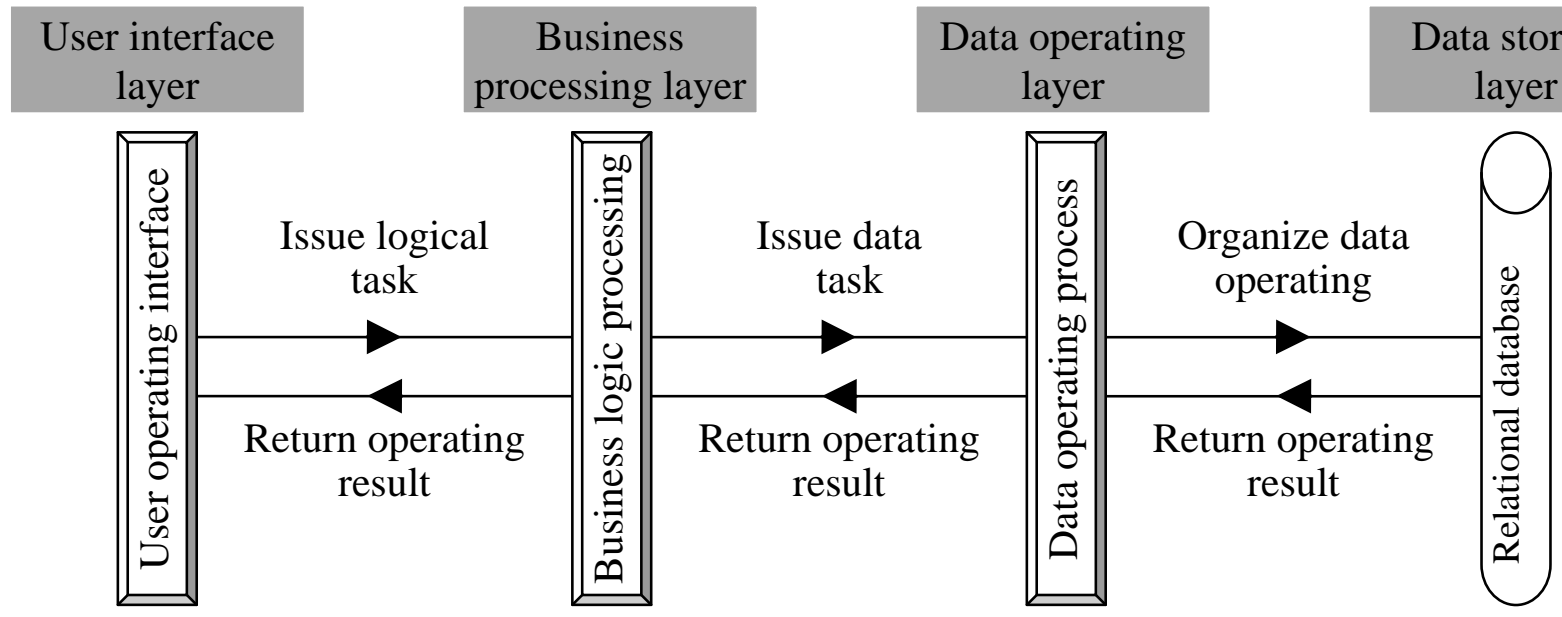

Fig. 1. Logical structure of hierarchical structure design

Fig. 1 shows the process of the multi layer logic structure as follows: the user layer is only responsible for the basic interface operation, and the operation is distributed to the corresponding business logic layer in the way of calling. The business logic layer is analyzed and processed according to the business logic. The data manipulation layer will organize the tasks to different data operations and interact with the database. The data operation layer returns the processed structure to the business logic layer, the business logic layer processes the received structure and then returns it to the user interface layer.

(2) Object oriented method. Object oriented method is a kind of systematic method to apply the idea of object oriented software development process to guide the development activities, which is based on the concept of "object". The object is composed of data package and allow operation, has a direct correspondence with the objective entity, a class defines a set of objects with similar properties, and inheritance is a way of attribute and operation of hierarchical relationships of share. The so-called object oriented is based on the concept of the object, the object as the center, the class and inheritance as the construction mechanism, understanding, understanding and characterization of the objective world, design, build the corresponding software system. The object oriented process is shown in Fig. 2.

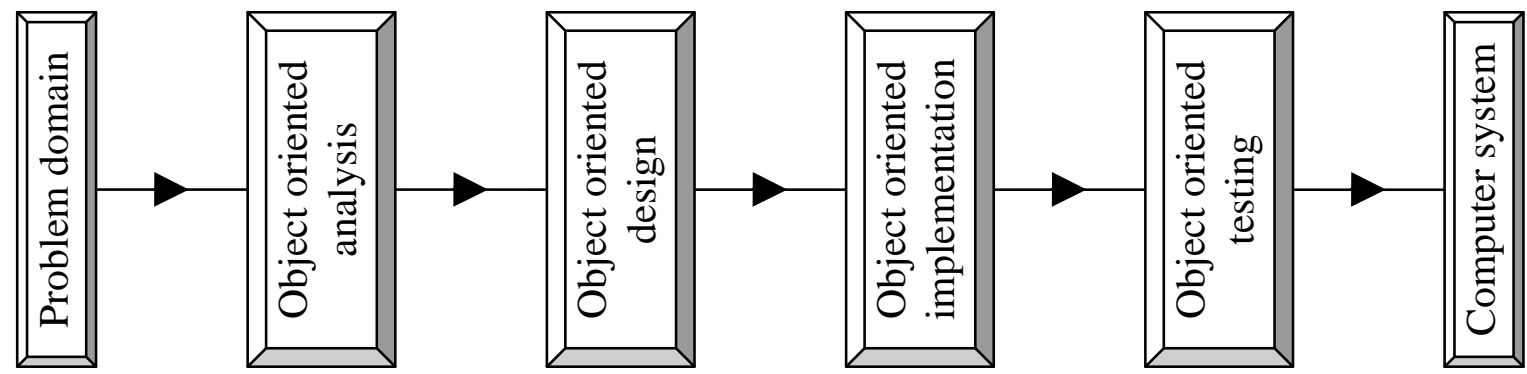

Fig. 2. Process of object oriented method

\section{System Function Design}

The function of the software is that the software should have the function and the function, the goal of the software should be expressed and realized through the function of the software. The functional design should follow the abstract, incremental refinement, modular system and 
information hiding principle. Effective modularity can be achieved by defining a set of mutually independent modules that communicate with each other using only the information necessary to implement the functionality of the software. By abstracting, you can identify the process entities that make up the software, and by hiding information, you can define and enforce access restrictions on the details of the module process and the local data structure. Using the principle of information concealment design software, testing and maintenance during the software to facilitate the work of the amendment. Because data and processes are hidden from other parts of the software, some of the unintended errors that are introduced are less likely to propagate to other parts of the software. Module independence is a direct product of concepts such as abstraction, modularity and information concealment. Module independence refers to each module in the software system has a single function, and not much contact with other modules.

English writing evaluation includes three ways [3,4], First, Automatic evaluation, the system automatically works on the student's grammar, syntax and text and other information to evaluate; Second, teacher evaluation, teachers of student works of language ability, pragmatic competence and writing level and other aspects of evaluation; Third, Mutual evaluation, students evaluate each other writing works, the evaluation process is the learning process. Follow the abstraction, step by step refinement, modularity and information concealment and other principles of the system function design results shown in Fig. 3.

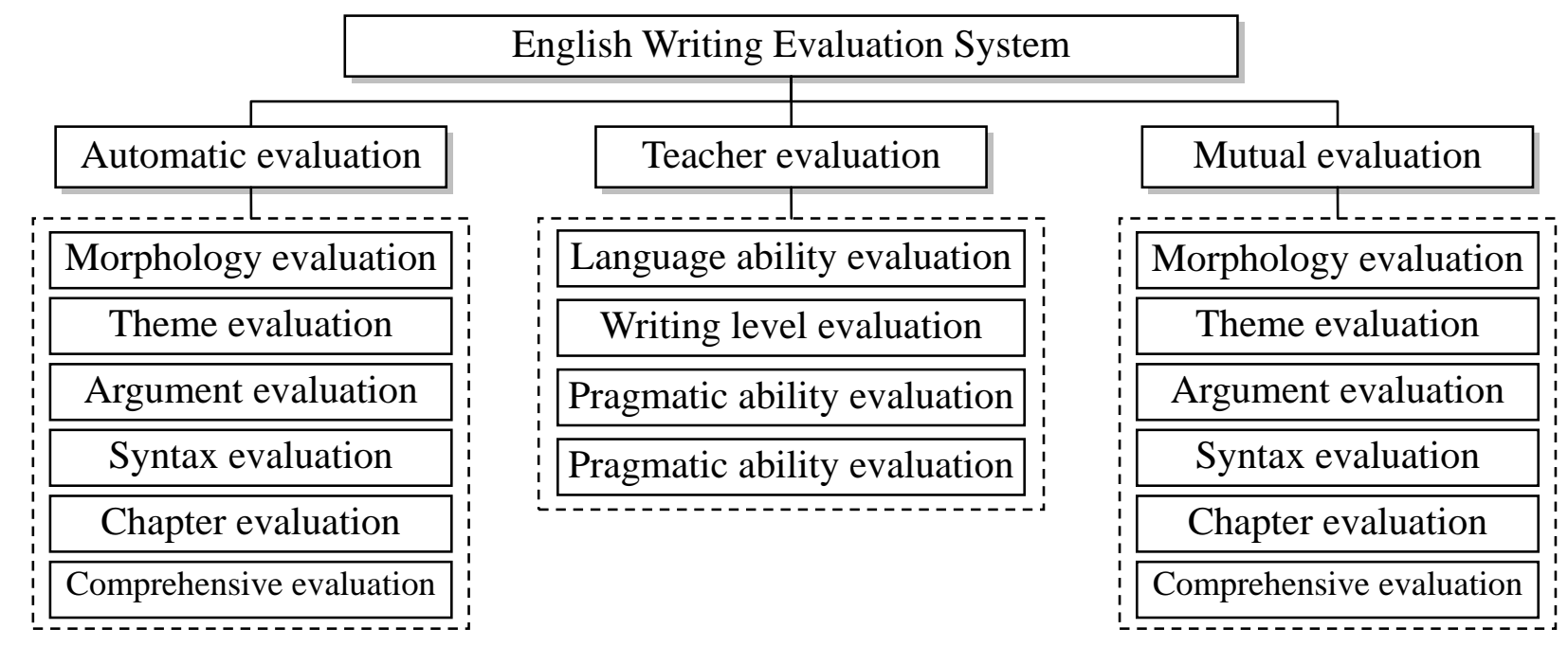

Fig. 3. Function on English Writing Evaluation System

\section{Key Technology of System Implementation}

Based on MS.NET development of English evaluation writing system involves three key technologies:

(1) User Interface Design. User interface is an important part of the application, mainly responsible for the user and the interaction between applications. The classes in the System.Windows.Forms name space can be divided into two categories: user interface controls in the design and running when displayed in the form, such as Label, Button, Textbox; non user interface controls, the design phase appeared in the special panel form, when the program is running is not visible, such as MenuStrip, Timer, etc.. In the design of the main form, for example, in the creation of the basic interface application of the project, the system automatically generates a form called Form1, rename it to frmMain, the property settings as shown in Table 1.

In order to facilitate the user to switch between multiple functions and use, the main form of the toolbar design, will be used in the form of buttons on the toolbar. Toolbar Toolbar controls, named imlToolbar, each button icon, with the ImageList control storage. After the main form interface design to add business processing code, including 5 steps: First, enter the main form of the code editing mode, add the name of the user to record the user name; Two, the main form loading event response program (frmMain_Load), and according to the log settings toolbar display items, open 
the system commonly used frmSite form; Three, click the toolbar event response program (tlbMain_ButtonClick), when you click on the toolbar to open a different sub form; The four is to determine how to display the need to open the form (HowShowFrm), according to the parameters of the incoming form name, the judge is to open a new form of the instance, or display the existing form instance; The five is to close the main form event response program (frmMain_Closing), close the form to request the user to confirm the operation; The six is to start the form and verify the identity of the user (main), the first to show the login form, requiring the user to verify the identity, if the login is successful, record the user permissions and display the main form.

Table 1. Project costs detail table

\begin{tabular}{c|c:c:c}
\hline Control name & Attribute name & Attribute value & Function description \\
\hline \multirow{4}{*}{ TrmMain } & $\begin{array}{c}\text { English Writing } \\
\text { Evaluation System }\end{array}$ & Set form title \\
\cline { 2 - 3 } & FormBorderStyle & Fixed3D & Set form border style \\
& Icon & Iscon\Sys.ico & Set form icon \\
\hline & IsMdiContainer & True & Can set the parent form \\
\hline
\end{tabular}

(2) Create data layer component. The data access component provides the caller with the means to perform the following tasks on the database: Create a record in the database, read the records in the database and return the business entity data to the caller, update the database with the modified business entity data provided by the caller In the record, delete the database records [5]. The methods for performing these four tasks are often referred to as the "CRUD" method, which is an acronym consisting of the initials of each task. The Data Access Logic component also provides a way to implement the business logic for a database. Often, a data access logic component accesses a single database and encapsulates data-related operations on a table or set of related tables in the database.

ADO.NET is a new data access technology proposed in the .NET Framework. The basic flow of accessing database is: Open the database by using the Connection data source in the data provider; use the Command or DataAdapter in the database provider to open the database Executes SQL commands; uses the DataReader dataset object DataSet in the data provider to read and process the data. ADO.NET access to the database process shown in Fig. 4.

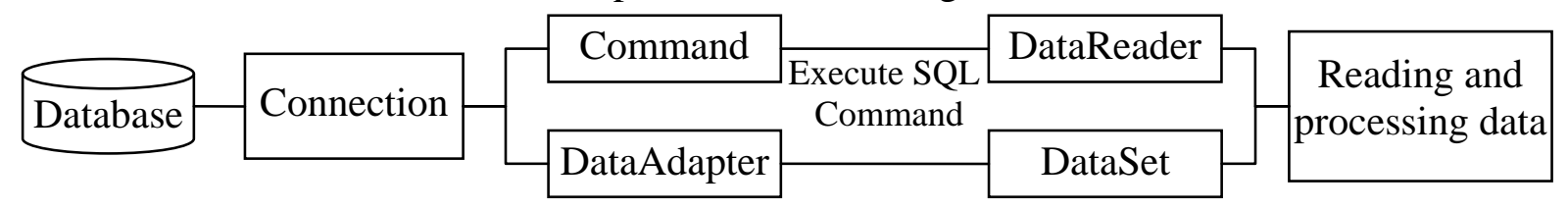

Fig. 4. Process of ADO.NET access to database

Database connection through the Connection object to achieve. Each data provider contains its own Connection object. Usually the database connection is: Declares a Connection object instance; assigns a ConnectionString property of the Connection object instance; calls the Connection object instance's Open () method to perform the connection. The key to the success of the connection is the ConnectionString value. There are different ConnectionStrings for different connection methods for different Connection objects. When the application and the database connection, you can read from the database table data, usually in two ways: one is to use the Command and DataReader objects, and the other is to use DataAdapter and DataSet objects. After you use the DataAdapter object to update the data and populate the DataSet, you can modify, add, and delete the data in the DataSet, and then update the data in the DataSet to the database.

(3) Create a Business Tier component. The understanding of business logic in a broad sense narrow sense. Generalized duty logic, is inherent in a software character, exists in the software product, is the software has a business logic in the field, is the core and soul of the software. Software products in addition to the interface and interaction of all can be seen as generalized 
business logic; narrow business logic, equivalent to hierarchical structure in the "business logic layer" of responsibility, is the software processing and business related tasks, the general narrow Of the business logic does not contain data persistence, but only concerned with the field of related business. This paper is designed for narrow business logic.

The business logic components separate the presentation layer and the data access layer to implement business rules. Create a business layer component of the process consists of five steps: First, create a business logic component classes, each class corresponds to a system database table, the method of each class for the completion of data retrieval, broadcast, update or delete operations , And apply the corresponding business rules [6]; Second, through the business logic component class to access Typed DataSets, you can use the Typed DataSet programming. Third, add field-level validation to the DataRow class, and field-level validation checks against the business object attribute values during an insert or update operation, as the business logic component class is added to the system. In order to improve security, the relevant rules in addition to the database level implementation, it should be implemented at the DataSet level; Fourth, business logic components to add custom business rules In addition to field-level validation, there may be advanced custom business rules, These rules refer to different entities, or to concepts that can not be expressed in a single column; Fifth, in the presentation layer to respond to validation errors, from the presentation layer call business logic layer components, you can deal with the possible exception, you can also throw exceptions directly to the .NET. Using the business logic component class to handle an exception, you can use the Try ... Catch block.

\section{Acknowledgement}

This work is supported by 2015 annual "Twelfth Five Year" planning project of Liaoning education and science (JG15DB026): Application Research on Automatic Scoring System in College English Writing.

\section{References}

[1] X. Y. Guo, "Diversified design for evaluation model of English writing," Journal of Beijing University of Posts and Telecommunications(Social Sciences Edition), vol. 13, no. 4, pp. 101-109, 2011.

[2] A. M. Chen, "A Multi-Perspective Approach to the Evaluation of English Writing," Journal of Liaoning Normal University(Social Science Edition), vol. 32, no. 6, pp. 107-109, 2009.

[3] X. P. Wu, "Review and development of network composition automatic scoring system," Educational information, vol. 57, no. 34, pp. 38-39, 2014.

[4] J. L. Tang, "How to integrate an automated writing assessment tool in the EFL classroom," Foreign Language Learning Theory and Practice, vol. 34, no. 1, pp. 49-57, 2014.

[5] Martin XJ's Blog, "Microsoft.NET data access layer component," http://www.cnblogs.com/martinxj/articles/28559.html, 2017-1-15.

[6] Chongqing Asia science and technology blog, "On the combination of.NET business logic layer," http://blog.sina.com.cn/s/blog_15b4a5c110102wxzu.html, 2017-1-15. 